\title{
Gadoxetic acid-enhanced magnetic resonance imaging: Hepatocellular carcinoma and mimickers
}

\author{
Yeun-Yoon Kim ${ }^{1,2}$, Mi-Suk Park ${ }^{1,2}$, Khalid Suliman Aljoqiman ${ }^{1,2,3}$, Jin-Young Choi ${ }^{1,2}$, and Myeong-Jin Kim ${ }^{1,2}$ \\ 'Department of Radiology, Severance Hospital, Yonsei University College of Medicine, Seoul; ${ }^{2}$ Research Institute of Radiological \\ Science, Yonsei University College of Medicine, Seoul, Korea; ${ }^{3}$ Department of Radiology, King Faisal University College of Medicine, \\ Al-Ahsa, Saudi Arabia
}

\begin{abstract}
Gadoxetic acid, a hepatocyte-specific magnetic resonance imaging (MRI) contrast agent, has emerged as an important tool for hepatocellular carcinoma (HCC) diagnosis. Gadoxetic acid-enhanced MRI is useful for the evaluation of earlystage HCC, diagnosis of HCC precursor lesions, and highly sensitive diagnosis of HCC. Furthermore, functional information provided by gadoxetic acid-enhanced MRI can aid in the characterization of focal liver lesions. For example, whereas lesions lack functioning hepatocytes appear hypointense in the hepatobiliary phase, preserved or enhanced expression of organic anion transporting polypeptides in some HCCs as well as focal nodular hyperplasia lead to hyperintensity in the hepatobiliary phase; and a targetoid appearance on transitional phase or hepatobiliary phase imaging can be helpful for identifying the histopathological composition of tumors. While gadoxetic acid-enhanced MRI may improve the sensitivity of HCC diagnosis and provide new insights into the characterization of focal liver lesions, there are many challenges associated with its use. This article reviews the pros and cons of HCC diagnosis with gadoxetic acidenhanced MRI and discuss some clues in the radiological differentiation of HCC from HCC mimickers. (Clin Mol Hepatol 2019;25:223-233)
\end{abstract}

Keywords: Diagnosis; Liver neoplasm; Hepatocellular carcinoma; Magnetic resonance imaging; Gadoxetic acid

\section{INTRODUCTION}

Liver cancer is the fourth leading cause of cancer-related death globally. Hepatocellular carcinoma (HCC) accounts for about $90 \%$ of primary liver cancers and its incidence is increasing worldwide. ${ }^{2}$ In patients with liver cirrhosis, a noninvasive diagnosis of HCC can be established by a radiological hallmark exhibited on dynamic liver protocol computed tomography (CT) or magnetic resonance imaging (MRI). ${ }^{2,3}$ The classic radiological hallmark is arterial phase hyperenhancement (APHE) and portal venous phase (PVP) or delayed phase "washout" on extracellular contrast agent (ECA)-enhanced CT or MRI.

During the past decade, hepatocyte-specific contrast agents for MRI have been recognized as important tools for the detection of small HCCs and the characterization of focal liver lesions. ${ }^{4-6} \mathrm{Ga}-$ doxetic acid (gadolinium-ethoxybenzyl-diethylenetriamine pentaacetic acid; Gd-EOB-DTPA; Primovist, Bayer Schering Pharma, Berlin, Germany), a widely used hepatocyte-specific contrast

\footnotetext{
Abbreviations:

ADC, apparent diffusion coefficient; APHE, arterial phase hyperenhancement CHCC-CCA, combined hepatocellular carcinoma-cholangiocarcinoma; DWI, diffusion-weighted imaging; ECA, extracellular contrast agent; $F N H$, focal nodular hyperplasia; HBP, hepatobiliary phase; HCA, hepatocellular adenoma; HCC, hepatocellular carcinoma; HGDN, high-grade dysplastic nodule; iCCA, intrahepatic mass-forming cholangiocarcinoma; LI-RADS, Liver Imaging Reporting and Data System; NHHN, non-hypervascular hepatobiliary phase hypointense nodule; OATP, organic anion transporting polypeptide; PVP, portal venous phase; TP, transitional phase
}

\section{Corresponding author : Mi-Suk Park}

Department of Radiology, Severance Hospital, Yonsei University College of Medicine, 50-1 Yonsei-ro, Seodaemun-gu, Seoul 03722, Korea

Tel: +82-2-2228-7400, Fax: +82-2-393-3035

E-mail: radpms@yuhs.ac

https://orcid.org/0000-0001-5817-2444 
agent, is excreted into the bile and urine at an approximate ratio of 1:1. The property of sufficient hepatobiliary excretion allows hepatobiliary phase (HBP) imaging, which is acquired approximately 20 minutes after contrast injection and exhibits excellent lesion-to-liver contrast.?

As the diagnosis of HCC at an early stage is desirable, the use of gadoxetic acid-enhanced MRI for HCC diagnosis is recommended by several guidelines, including the Asia-Pacific clinical practice guidelines, the Japan Society of Hepatology guidelines, and the Korean Liver Cancer Study Group-National Cancer Center Korea Practice guidelines. ${ }^{8-10}$ The Liver Imaging Reporting and Data System (LI-RADS) has also embraced the use of gadoxetic acidenhanced MRI since 2014. ${ }^{11}$ The performance of HCC diagnostic criteria in gadoxetic acid-enhanced MRI is under continuous investigation..$^{12-16}$

This article first reviews the pros and cons of a radiological diagnosis of HCC with gadoxetic acid-enhanced MRI. Thereafter, some clues in the radiological differentiation of HCC from HCC mimickers will be discussed. Mimickers of HCC to be differentiated by imaging include 1) non-HCC malignancies, such as intrahepatic mass-forming cholangiocarcinoma (iCCA), combined hepatocellular carcinoma-cholangiocarcinoma (CHCC-CCA), and hypervascular metastasis; 2) benign hepatocellular lesions, including focal nodular hyperplasia (FNH), FNH-like nodules, and hepatocellular adenoma (HCA); and 3) benign non-hepatocellular lesions, such as hemangioma, hypervascular pseudolesions, and angiomyolipoma.

\section{HCC DIAGNOSIS USING GADOXETIC ACID- ENHANCED MRI}

The major advantage of using gadoxetic acid-enhanced MRI is the improved detection of small $(\leq 2 \mathrm{~cm}) \mathrm{HCCs}$ compared with ECA-enhanced MRI or CT. ${ }^{17-19}$ The detection sensitivity can be further heightened by the use of diffusion-weighted imaging (DWI). ${ }^{20}$ Furthermore, it has been demonstrated that the combined use of gadoxetic acid-enhanced MRI and CT can diagnose additional HCCs compared with the use of $\mathrm{CT}$ alone, which may change the tumor stage and lead to improved survival outcomes. ${ }^{21}$ Therefore, gadoxetic acid-enhanced MRI is considered an important diagnostic tool, particularly in patients with early-stage HCC.

Another advantage of using gadoxetic acid-enhanced MRI is that it facilitates the diagnosis of borderline hepatic nodules (i.e., early HCC and high-grade dysplastic nodule [HGDN]), which are considered precursors of progressed, distinctly nodular HCC. ${ }^{12,22}$ Early HCC is histopathologically defined as vaguely nodular, small $(\leq 2 \mathrm{~cm})$, well-differentiated HCC and is distinguished from HGDNs in terms of the presence of stromal invasion. ${ }^{23}$ While progressed HCCs are typically hypervascular on imaging, early HCCs rarely exhibit arterial hypervascularity and thus are not diagnosed as HCC by this radiological hallmark. ${ }^{24}$ On the other hand, the expression of organic anion transporting polypeptide 1B3 (OAT$\mathrm{P} 1 \mathrm{~B} 3)$, the transporter of gadoxetic acid, starts decreasing prior to neo-arterialization during hepatocarcinogenesis. ${ }^{24,25}$ Consequently, early HCCs and approximately one-third of HGDNs appear as non-hypervascular HBP hypointense nodules (NHHNs) on gadoxetic acid-enhanced MRI, contrary to benign cirrhosis-associated nodules or low-grade dysplastic nodules which are usually isointense in the HBP due to preserved OATP1B3 expression. ${ }^{26,27}$ About one-third of NHHNs undergo hypervascular transformation within 3 years. ${ }^{28}$ In addition, the presence of an NHHN is regarded as a cause of intrahepatic recurrence after surgical resection or radiofrequency ablation. ${ }^{29,30}$ The management strategy for these borderline lesions is not yet established and therefore needs further investigation. $^{31}$

Concerns have been raised regarding the decrease in the specificity of HCC diagnosis when using gadoxetic acid-enhanced MRI. ${ }^{31}$ The radiological hallmark (i.e., APHE and PVP or delayed phase "washout") used in ECA-enhanced CT or MRI provides lower specificity on gadoxetic acid-enhanced MRI, which can be attributed to the "pseudo-washout" phenomenon observed in HCC mimickers (i.e., the relative hypointensity of a non-HCC lesion in the transitional phase [TP] due to adjacent liver parenchymal contrast uptake)., ${ }^{523-34}$ Therefore, the 2018 European Association for the Study of the Liver guidelines and LI-RADS require that "washout" be assessed only in the PVP when using gadoxetic acid-enhanced MRI, not in the TP or HBP. 2,35 However, PVP "washout" in gadoxetic acid-enhanced MRI has been regarded as less sensitive for HCC than TP or HBP hypointensity, especially for small HCCS, and is not independently predictive of $\mathrm{HCC}^{12,13}$ Studies have also demonstrated that use of the HBP improves the detection of small HCCs and provides near-perfect sensitivity for HCC diagnosis. ${ }^{13,36}$

Recent studies have suggested that the exclusion of HCC mimickers may overcome the hurdle of "pseudo-washout" and enable a more sensitive diagnosis of HCC on gadoxetic acid-enhanced MRI. ${ }^{12-14,37}$ When benign lesions, such as hemangioma (i.e., LIRADS category 1 or 2) and possible non-HCC malignancy (i.e., LIRADS category M) are properly excluded by the LI-RADS algo- 
rithm, an evaluation of "washout" in the TP as well as the PVP can diagnose HCC without impairing specificity (Fig. 1). ${ }^{37}$ Furthermore, the evaluation of "washout" in the PVP, TP, or HBP in lesions that are not LI-RADS category 1, 2, or M identifies HCC with acceptable specificity. ${ }^{14}$ When hemangiomas are excluded from nodules detected by surveillance ultrasound, the presence of APHE and HBP hypointensity can provide comparable specificity to that of APHE and PVP or TP hypointensity; the satisfaction of APHE, HBP hypointensity, and restricted diffusion criteria can result in similar specificity to that of APHE and PVP "washout" criteria. ${ }^{12,13}$ These diagnostic criteria may be especially applicable when improved sensitivity for HCC diagnosis is prioritized over an "acceptable" decrease in specificity. ${ }^{31}$

\section{MIMICKERS OF HCC}

\section{Non-HCC malignancy}

Non-HCC malignancy frequently exhibits a targetoid appearance on imaging, namely targetoid dynamic enhancement, targetoid diffusion restriction, and a targetoid TP or HBP appearance. ${ }^{35}$ The appearance results from the histopathological composition of the tumor with dense fibrous stroma at the core and hypercellularity at the periphery. The peripheral cellular component shows hyperenhancement in the arterial phase and hypoenhancement in the following phases, while the central fibrous stroma enhances progressively throughout dynamic imaging. A targetoid appearance on TP or HBP imaging as observed on gadoxetic acid-enhanced MRI refers to mild contrast retention in the fibrous core and a lack of contrast uptake in the peripheral cellular portions; the feature can sometimes be the sole identifier of an iCCA on imaging. ${ }^{37,38}$ Pitfalls in radiological differentiation between nonHCC malignancy and HCC lie in the facts that HCC with atypical imaging features can exhibit targetoid features as well (Fig. 2) and that some non-HCC malignancies, especially CHCC-CCA and small-sized iCCAs, can mimic HCC by demonstrating a non-targetoid appearance.

Similar to ECA-enhanced MRI, rim APHE, being a targetoid feature, is the most important imaging feature distinguishing iCCA from HCC on gadoxetic acid-enhanced MRI. ${ }^{33,39,40}$ However, HCC with abundant fibrotic stroma or a central scar can also be observed as a targetoid mass. ${ }^{41}$ Features of the enhancing "capsule," an intratumoral septum, and multifocal T2 hyperintense foci can be clues for identifying HCC when it mimics iCCA on imaging. ${ }^{41,42}$ Owing to the overlaps in targetoid features among HCC and nonHCC malignancies, histopathologic confirmation can be considered for a targetoid mass. ${ }^{3}$ On the other hand, iCCA can also depict non-rim APHE, particularly when the tumor is small in size. ${ }^{39,43}$ Histopathologically, "small duct type" iCCAs arise commonly from the peripheral small bile duct in a cirrhotic liver or chronic hepatitis and have a tendency to possess less stromal fibrotic tissue than "large duct type" iCCAs, which originate from chronically diseased perihilar bile ducts. ${ }^{44,45}$ Because of their histopathologic characteristics, "small duct type" iCCAs depict a larger area of APHE and diffusion restriction on imaging and thus are likely to mimic HCC (Fig. 3). ${ }^{46}$

The discrimination of CHCC-CCA from HCC by imaging has also been considered challenging. The histopathologic components of cHCC-CCA are presumed to determine its radiological appear-
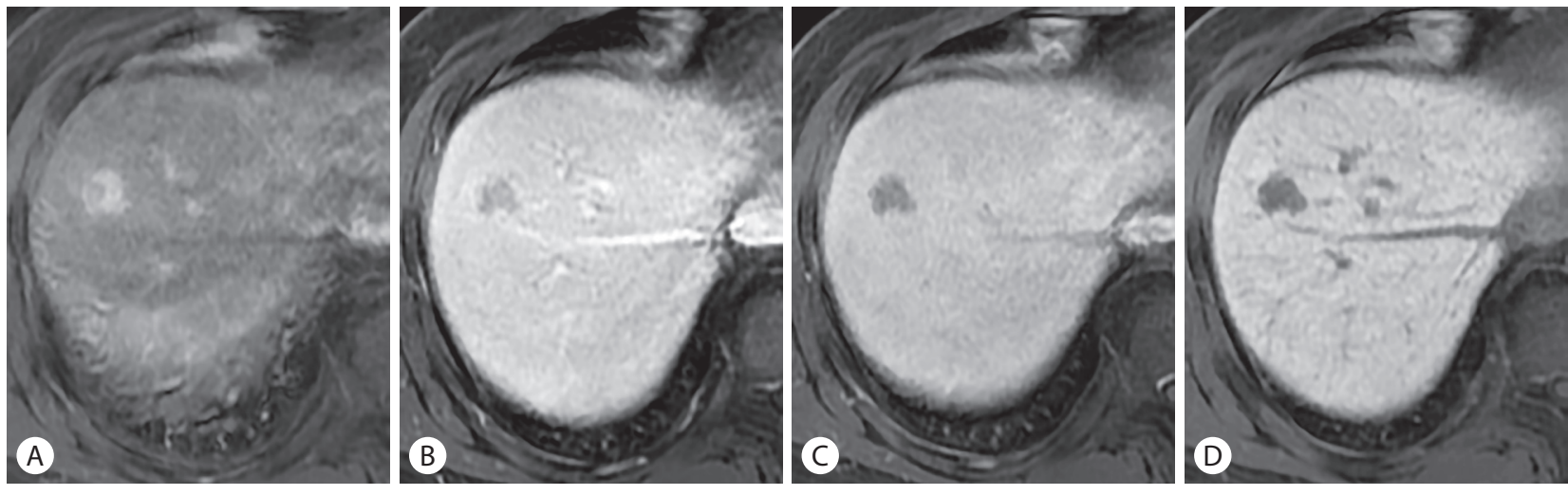

Figure 1. A small hepatocellular carcinoma (HCC) in a 58-year-old man with chronic hepatitis B. A 19-mm nodule in hepatic segment VIII depicts (A) non-rim arterial phase hyperenhancement and "washout" in the (B) portal venous phase, qualifying it as Liver Imaging Reporting and Data System category 5 (i.e., definite HCC). Hypointensity in the (C) transitional phase and (D) hepatobiliary phase are also noted on gadoxetic acid-enhanced magnetic resonance imaging. 
ance. ${ }^{47}$ While CHCC-CCA with predominant cholangiocarcinoma components is more likely to demonstrate a targetoid mass, cHCC-CCA with predominant HCC components is more likely to mimic HCC by showing non-rim APHE or a strong degree of rim APHE (Fig. 4). ${ }^{48,49}$ Furthermore, the combination of non-rim APHE and PVP "washout" is not infrequently observed among CHCC-
CCAs, especially among tumors smaller than $2 \mathrm{~cm}^{50}$ As the histopathological terminology for $\mathrm{CHCC}-\mathrm{CCA}$ has been recently re-defined by expert consensus, ${ }^{47}$ future studies are warranted to elucidate the radiological and clinical characteristics of CHCC-CCA in a large-scale cohort.

The possibility of hypervascular metastasis needs to be priori-
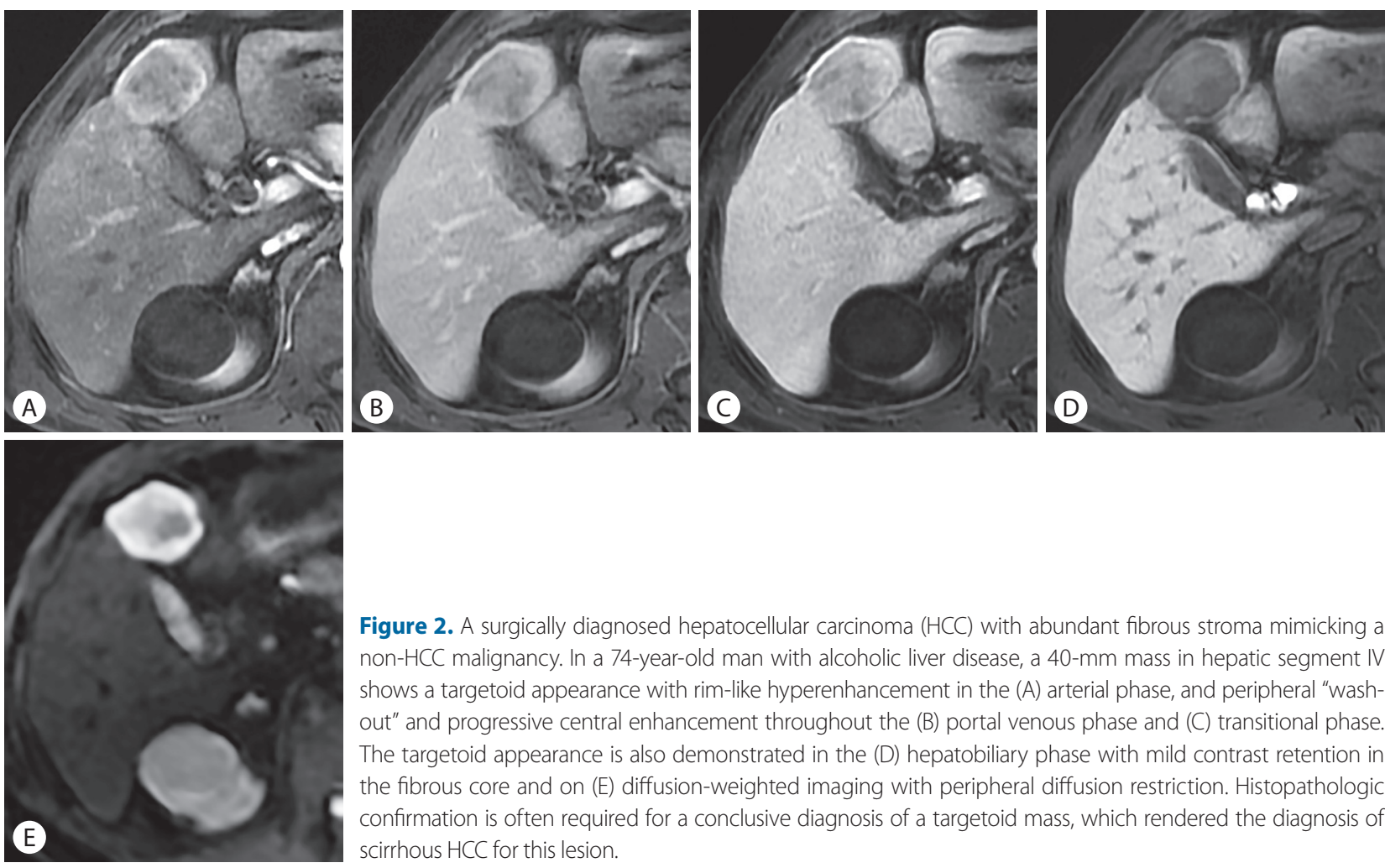

Figure 2. A surgically diagnosed hepatocellular carcinoma (HCC) with abundant fibrous stroma mimicking a non-HCC malignancy. In a 74-year-old man with alcoholic liver disease, a 40-mm mass in hepatic segment IV shows a targetoid appearance with rim-like hyperenhancement in the (A) arterial phase, and peripheral "washout" and progressive central enhancement throughout the (B) portal venous phase and (C) transitional phase. The targetoid appearance is also demonstrated in the (D) hepatobiliary phase with mild contrast retention in the fibrous core and on (E) diffusion-weighted imaging with peripheral diffusion restriction. Histopathologic confirmation is often required for a conclusive diagnosis of a targetoid mass, which rendered the diagnosis of scirrhous HCC for this lesion.
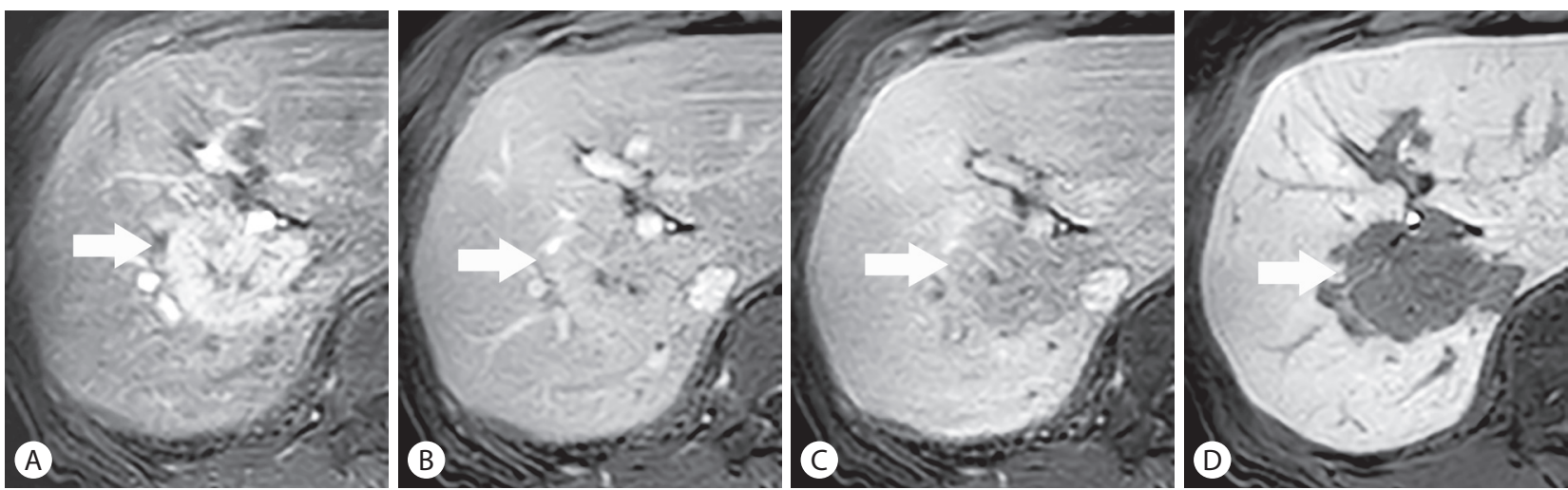

Figure 3. An intrahepatic mass-forming cholangiocarcinoma (iCCA) mimicking hepatocellular carcinoma (HCC) in a 79-year-old woman with no underlying liver disease. A 46-mm lobulated mass (arrows) in hepatic segment VII shows non-rim hyperenhancement in the (A) arterial phase and persistent contrast enhancement in the (B) portal venous phase. The nodule depicts mild hypointensity in the (C) transitional phase (i.e., "pseudo-washout") and hypointensity in the (D) hepatobiliary phase from background liver parenchymal enhancement. The histopathological examination after surgical resection revealed a diagnosis of small duct type iCCA, which is likely to mimic HCC on imaging. 
Yeun-Yoon Kim, et al.

tized over that of HCC when a patient has an active extrahepatic malignancy and no underlying liver disease. Neuroendocrine tumor, renal cell carcinoma, choriocarcinoma, melanoma, breast cancer, thyroid cancer, and other hypervascular tumors could metastasize to the liver. ${ }^{51}$ On the contrary, when an active extrahepatic malignancy and liver cirrhosis are present at the same time, radiological clues indicating $\mathrm{HCC}$, such as intratumoral fat (Fig. 5) or blood products, may properly prompt a histopathologic confirmation of the liver mass.

\section{Benign hepatocellular lesions}

Gadoxetic acid-enhanced MRI can help characterize benign hyperplastic hepatocellular lesions, such as FNH in non-cirrhotic liver and an FNH-like nodule in cirrhotic liver, which can be managed conservatively. These lesions, consisting of hyperplastic hepatocytes, typically appear iso- or hyperintense in the HBP with a central hypointense scar and lobulated margins. ${ }^{26}$ However, because of the hypervascular nature of FNH or an FNH-like nodule, these
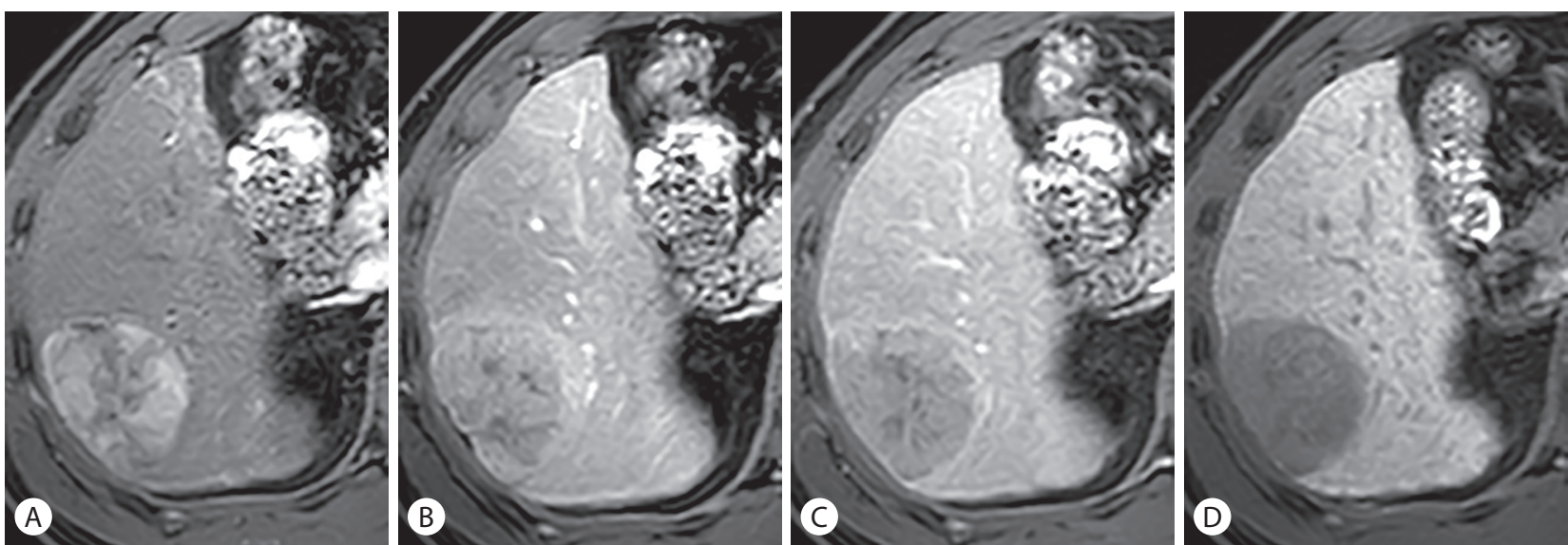

Figure 4. A combined hepatocellular carcinoma-cholangiocarcinoma (CHCC-CCA) mimicking hepatocellular carcinoma (HCC) in a 49-year-old man with hepatitis B-related liver cirrhosis. A 56-mm mass in hepatic segment VI depicts non-rim hyperenhancement in the (A) arterial phase. Partially hypointense areas are noted in the (B) portal venous phase, suggesting the presence of "washout." A hyperintense rim around the mass is noted in the (C) transitional phase, suggesting the presence of an enhancing "capsule." The nodule appears as a diffusely hypointense mass in the (D) hepatobiliary phase. The histopathological examination after surgical resection revealed CHCC-CCA with an HCC component comprising $80 \%$ of the mass. The cholangiocarcinoma component comprising $20 \%$ of the mass is not well appreciated on imaging.
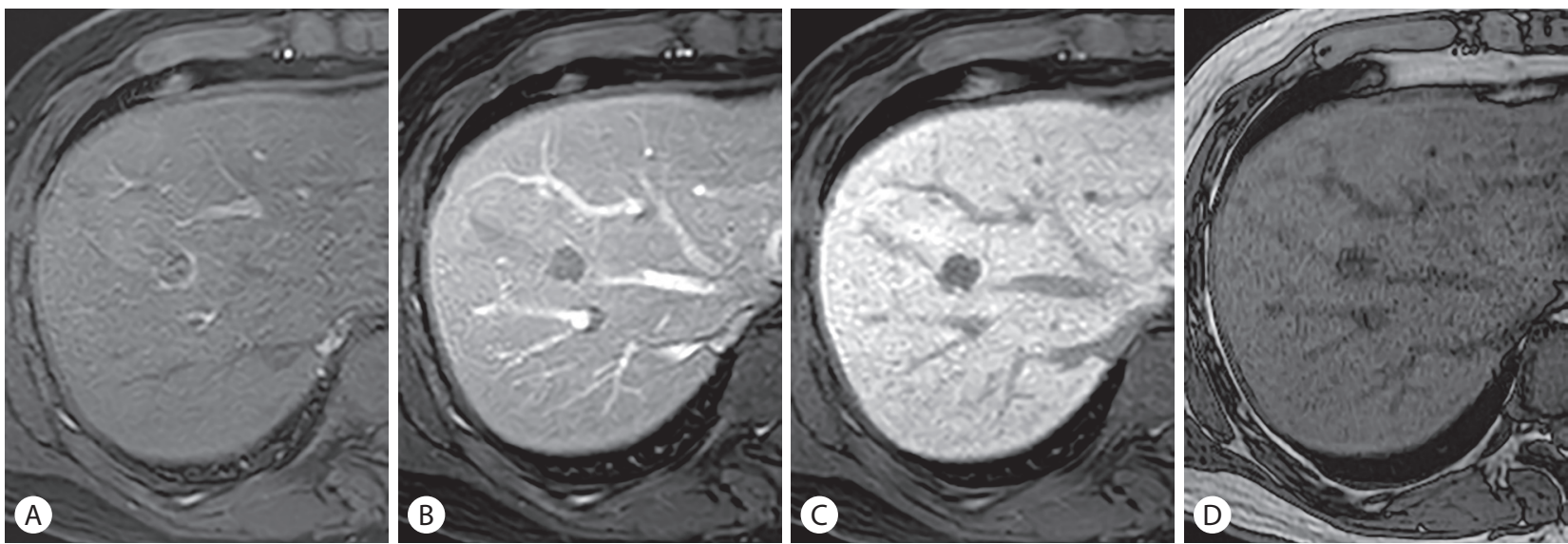

Figure 5. A surgically diagnosed hepatocellular carcinoma (HCC) in a 53-year-old man with a history of rectal cancer and hepatitis B-related liver cirrhosis. A 19-mm nodule in hepatic segment IV/VIII demonstrates rim-like hyperenhancement in the (A) arterial phase, and diffuse hypointensity in the (B) portal venous phase and (C) hepatobiliary phase. (D) The out-of-phase image depicts diffuse hypointensity of the lesion, suggesting the presence of a fat component. Although rim arterial phase hyperenhancement can be seen in metastasis from rectal cancer, the presence of a fatty component and liver cirrhosis favors the diagnosis of HCC over metastasis and necessitates a histopathological diagnosis. 


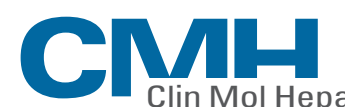

Volume 25 Number 3 September 2019
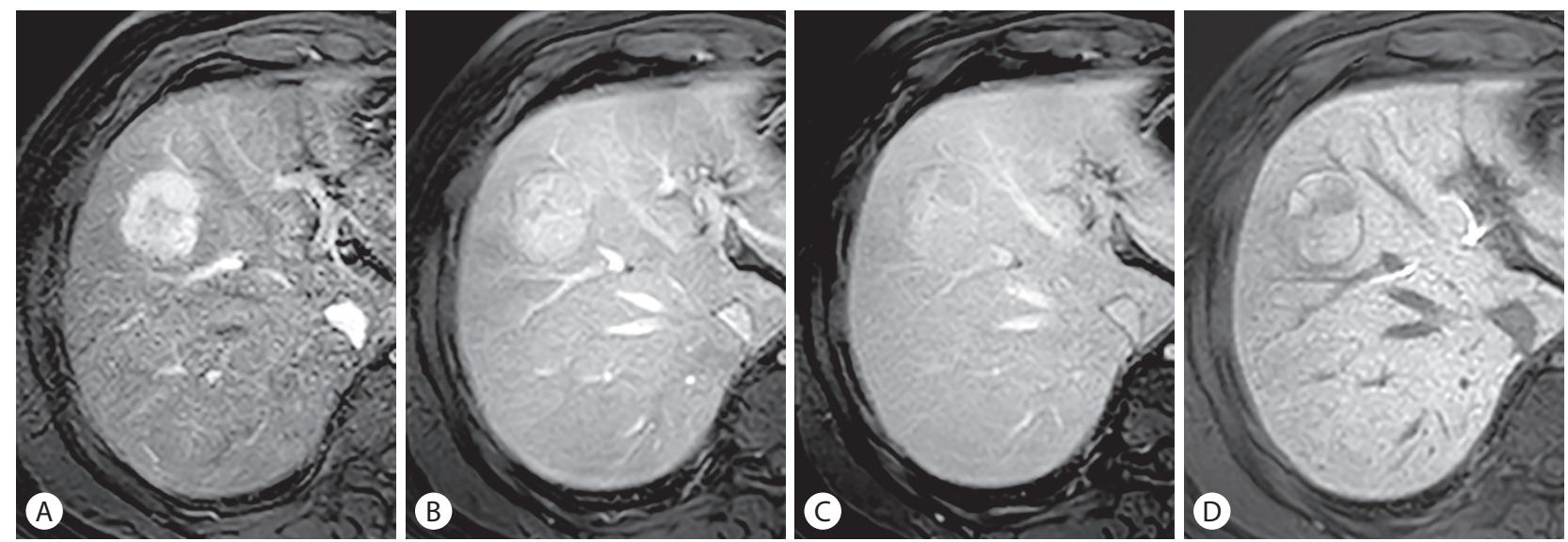

Figure 6. Hepatocellular carcinoma (HCC) with preserved organic anion transporting polypeptide expression in a 60-year-old man with chronic hepatitis B. A 35-mm mass in hepatic segment VIII shows (A) non-rim arterial phase hyperenhancement and persistent enhancement throughout the (B) portal venous phase, (C) transitional phase, and (D) hepatobiliary phase (HBP), showing iso- to hyperintensity to the adjacent liver. The smooth, hypointense rim shown in the (D) HBP favors the diagnosis of HCC over that of a benign lesion. The histopathological examination after surgical resection revealed Edmondson grade I to II differentiation of the tumor.
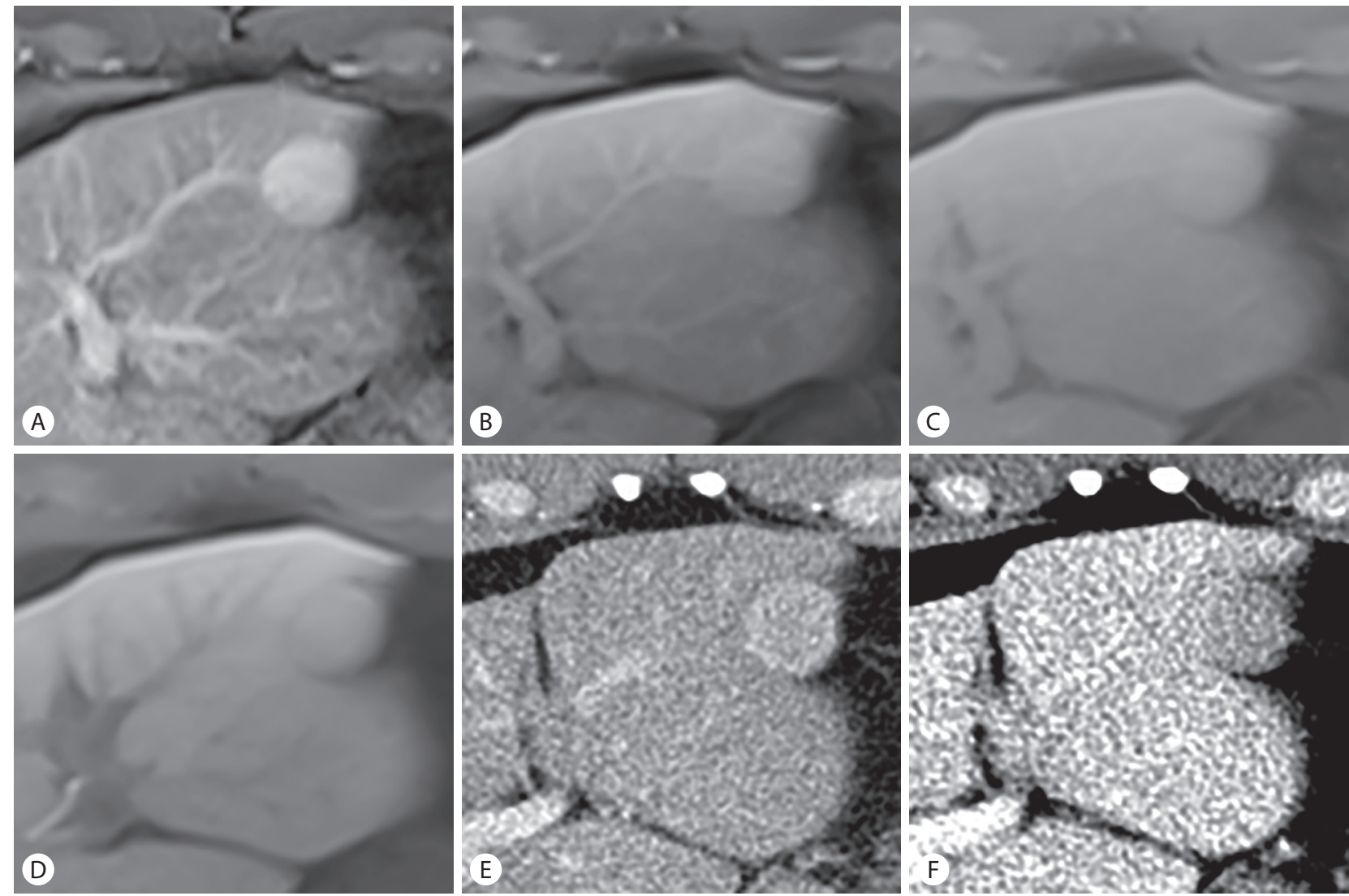

Figure 7. Hepatocellular adenoma with $\beta$-catenin mutation in a 48-year-old man with no underlying liver disease. A 21-mm nodule in hepatic segment II depicts non-rim arterial phase hyperenhancement on (A) gadoxetic acid-enhanced magnetic resonance imaging (MRI). Persistent enhancement is demonstrated throughout the (B) portal venous phase (PVP), (C) transitional phase, and (D) hepatobiliary phase, which is a typical enhancement pattern for the $\beta$-catenin-activated subtype. The nodule also shows non-rim arterial phase hyperenhancement on (E) computed tomography (CT) image. However, contrary to the finding on MRI, the nodule shows hypoenhancement in the (F) PVP of CT. 
lesions can resemble HCC; FNH-like nodules which are frequently observed in patients with alcoholic liver cirrhosis can be misinter- preted as HCCs. Furthermore, about $5 \%$ to $20 \%$ of HCCs have preserved or enhanced OATP1B3 expression and appear iso- or
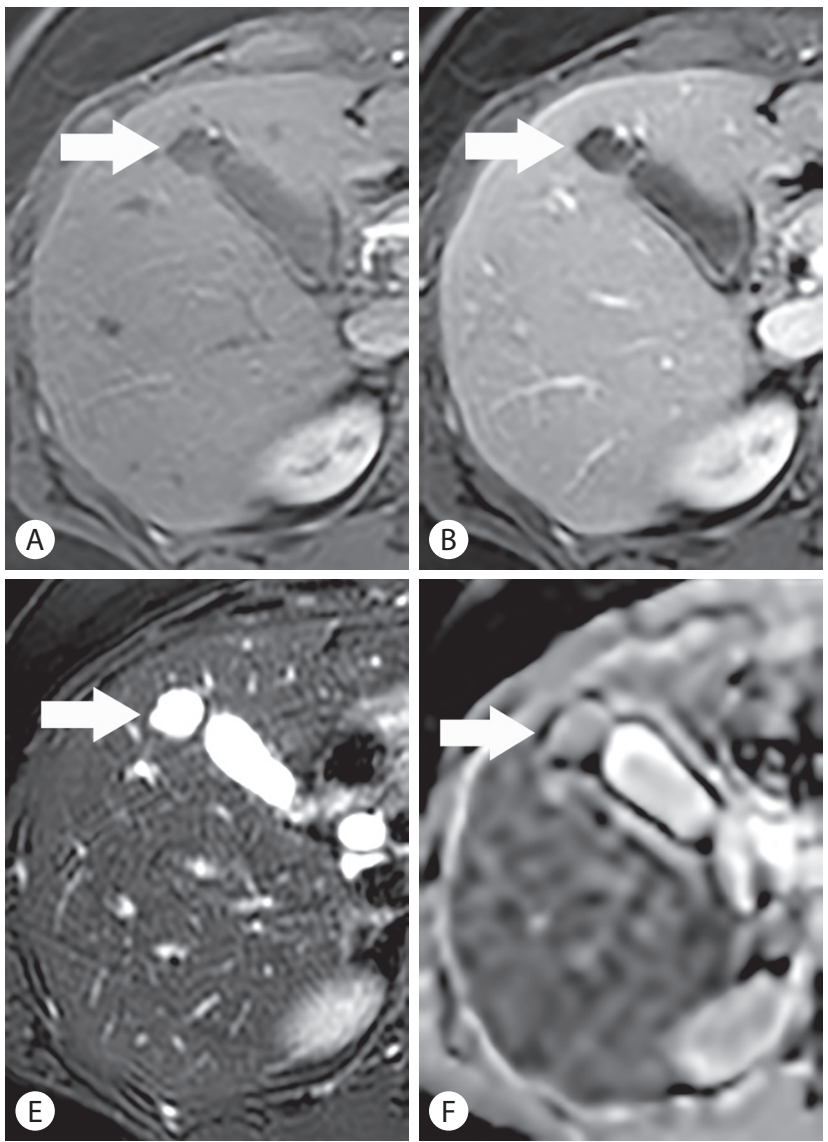
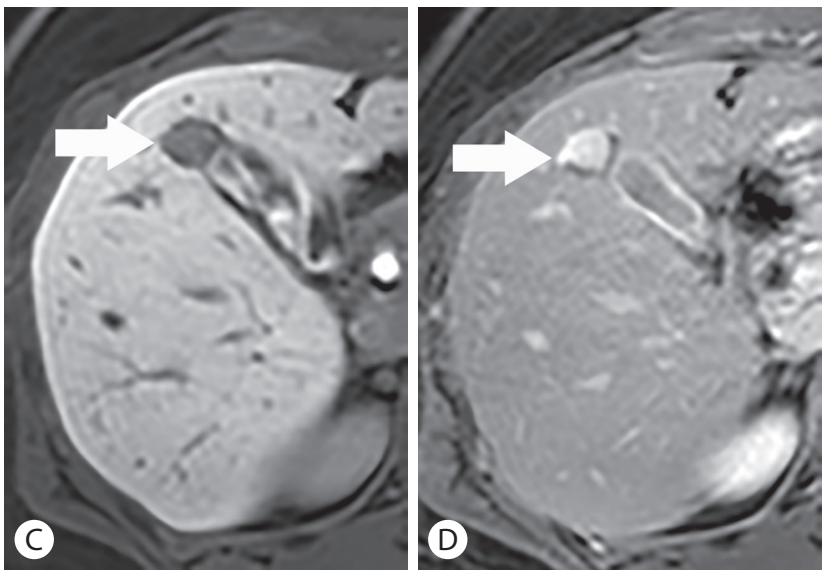

Figure 8. Discrepant imaging features of hemangioma according to the magnetic resonance imaging (MRI) contrast media used. A 14-mm nodule (arrows) was incidentally found in hepatic segment IV/V in a 45-year-old woman. On both gadoxetic acid-enhanced MRI and extracellular contrast agent (ECA)-enhanced MRI (not shown), the nodule shows peripheral nodular enhancement in the (A) arterial phase and slight progressive enhancement along the lesion periphery in the (B) portal venous phase, which are indicative of a slowly enhancing hemangioma. The nodule appears as a defect in contrast uptake in the (C) hepatobiliary phase due to a lack of functioning hepatocytes, while diffuse contrast enhancement is demonstrated in the (D) delayed phase of ECA-enhanced MRI as a result of its prominent vasculature. (E) Bright signal intensity in a heavily T2weighted image and (F) a high apparent diffusion coefficient value are suggestive of hemangioma.
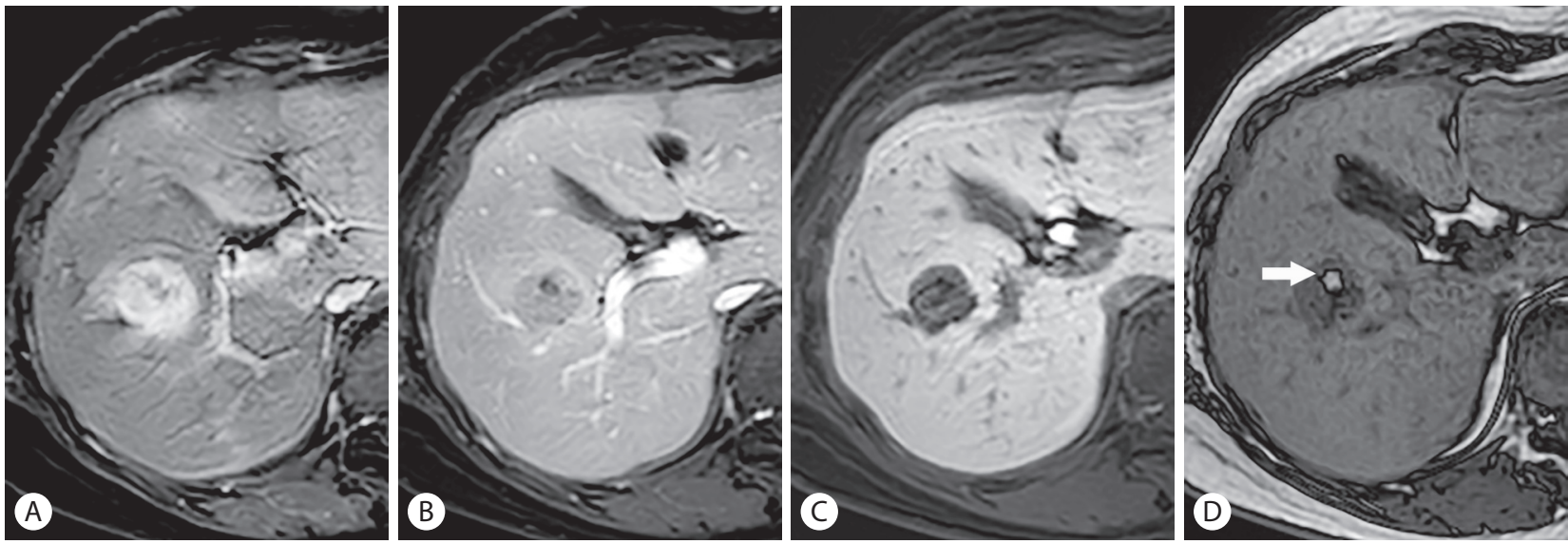

Figure 9. Biopsy-proven angiomyolipoma in a 55-year-old woman with no underlying liver disease. A 27-mm mass in hepatic segment $V$ shows nonrim hyperenhancement in the (A) arterial phase and mild hypointensity in the (B) portal venous phase, mimicking hepatocellular carcinoma (HCC). Marked and homogeneous hypointensity in the (C) hepatobiliary phase may favor a diagnosis of angiomyolipoma over that of HCC. (D) The out-ofphase image depicts diffuse hypointensity of the lesion, suggesting the presence of a fat component; a small, hyperintense, fatty focus (arrow) appears hypointense on other fat-suppressed images (A-C). As both angiomyolipoma and HCC possibly contain intratumoral fat and are hypervascular, the exact radiological differentiation remains challenging. 
hyperintense in the HBP, mimicking FNH (Fig. 6). ${ }^{25,52}$ Moreover, these HCCs frequently do not exhibit PVP or TP hypointensity, similar to $\mathrm{FNH}$, because of active contrast uptake by the tumor. ${ }^{25}$ The presence of HBP hypointense foci or a smooth hypointense rim in the HBP are features differentiating HCC from FNH or benign cirrhosis-associated nodules. ${ }^{53}$ In addition, dynamic CT can be helpful in the differentiation by depicting "washout" of HCC in the PVP or delayed phase regardless of the OATP1B3 expression level. ${ }^{54}$

Gadoxetic acid-enhanced MRI can aid in the characterization of HCA, especially for the subtyping. ${ }^{26,55,56}$ On HBP imaging, about $75 \%$ of HCAs appear hypointense to the adjacent liver, which is useful for differentiation of these tumors from $\mathrm{FNH}$; hepatocyte nuclear factor 1 alpha-inactivated HCAs, most inflammatory HCAs, and unclassified HCAs are characterized by HBP hypointensity. ${ }^{26,55}$ On the other hand, $\beta$-catenin-activated HCAs and some inflammatory HCAs with $\beta$-catenin mutation may exhibit HBP hyperintensity primarily due to OATP1B3 overexpression (Fig. 7). ${ }^{26,56}$ The risk of malignant transformation is high for HCA with $\beta$-catenin mutation, which may merit surgical resection. ${ }^{55} \mathrm{MRI}$ can also help in HCA subtyping by depicting the fat components of hepatocyte nuclear factor 1 alpha-inactivated HCA as well as the telangiectatic features of inflammatory HCA as a T2 high-signal area in the lesion periphery (i.e., the atoll sign). ${ }^{56}$

\section{Benign non-hepatocellular lesions}

Hemangioma, the most common benign tumor of the liver, needs to be differentiated from HCC. The phenomenon of "pseudo-washout" can make the differentiation difficult on gadoxetic acid-enhanced MRI (Fig. 8), especially for rapidly enhancing or high-flow hemangioma. ${ }^{57,58}$ Nonetheless, markedly bright signal intensity comparable to that of the cerebrospinal fluid on a heavily T2-weighted image (a fluid-sensitive protocol with a long echo time), a high apparent diffusion coefficient (ADC) value, and signal intensity equivalent to that of the portal vein suggests hemangioma. ${ }^{59-61}$

Hypervascular pseudolesions or arterioportal shunts are frequently observed in cirrhotic liver, mimicking $\mathrm{HCC}^{62}$ The differentiation of these findings from $\mathrm{HCC}$ can be aided greatly by the HBP of gadoxetic acid-enhanced MRI. Hypervascular pseudolesions are typically wedge-shaped and found in a subcapsular location, while some are nodular in appearance. ${ }^{6}$ The majority of hypervascular pseudolesions are isointense or only slightly hypointense in the HBP, contrary to HCCs, which are generally hypoin- tense in the $\mathrm{HBP}^{63,64} \mathrm{~A}$ lack of hypointensity in the HBP combined with isointensity on DWI can properly identify hypervascular pseudolesions. $^{63}$

The radiological distinction between angiomyolipoma and HCC is challenging because of overlapping imaging features, such as the contrast enhancement profile (APHE and PVP or TP hypointensity), diffusion restriction, and intratumoral fat (Fig. 9).6,65,66 This differentiation is particularly problematic in non-cirrhotic liver and commonly requires histopathologic proof. On gadoxetic acidenhanced MRI, the hypointensity of angiomyolipoma in the HBP tends to be marked and homogeneous compared to that of HCC. ${ }^{65,66}$ This can be attributed to a complete lack of hepatocytes in angiomyolipoma in contrast to $\mathrm{HCC}$, which may contain dysplastic foci. In addition, persistent enhancement in the PVP, presence of an early draining vein, and absence of an enhancing "capsule" favor the diagnosis of angiomyolipoma over $\mathrm{HCC} .{ }^{65}$ Last but not least, angiomyolipoma tends to occur more frequently in female patients, patients younger in age, and in normal liver. ${ }^{65,66}$

\section{CONCLUSIONS}

The strengths of using gadoxetic acid-enhanced MRI include the facilitated diagnosis of small HCCs and precursor lesions of progressed HCC. The exclusion of HCC mimickers may help overcome the diagnostic hurdle of "pseudo-washout" on gadoxetic acid-enhanced MRI and enable the highly sensitive diagnosis of HCC without significant specificity impairment. The differentiation of HCC from HCC mimickers can be aided by functional information provided by gadoxetic acid. However, pitfalls in the characterization of focal liver lesions unique to gadoxetic acid-enhanced MRI needs to be recognized for a more accurate diagnosis of HCC.

\section{Authors' contribution}

YY Kim and MS Park contributed to this paper with conception and design of the study; YY Kim and KS Aljogiman performed literature review and drafting; MS Park, JY Choi, and MJ Kim performed critical revision and editing; All authors approved the final version of the manuscript.

\section{Conflicts of Interest}

The authors have no conflicts of interest to disclose. 


\section{REFERENCES}

1. Global Burden of Disease Liver Cancer Collaboration. The burden of primary liver cancer and underlying etiologies from 1990 to 2015 at the global, regional, and national level: results from the global burden of disease study 2015. JAMA Oncol 2017;3:1683-1691.

2. European Association for the Study of the Liver. EASL clinical practice guidelines: management of hepatocellular carcinoma. J Hepatol 2018;69:182-236.

3. Marrero JA, Kulik LM, Sirlin CB, Zhu AX, Finn RS, Abecassis MM, et al. Diagnosis, staging, and management of hepatocellular carcinoma: 2018 practice guidance by the American Association for the Study of Liver Diseases. Hepatology 2018;68:723-750.

4. Joo I, Lee JM. Recent advances in the imaging diagnosis of hepatocellular carcinoma: value of gadoxetic acid-enhanced MRI. Liver Cancer 2016;5:67-87.

5. Choi JY, Lee JM, Sirlin CB. CT and MR imaging diagnosis and staging of hepatocellular carcinoma: part II. extracellular agents, hepatobiliary agents, and ancillary imaging features. Radiology 2014;273:3050.

6. Park YS, Lee CH, Kim JW, Shin S, Park CM. Differentiation of hepatocellular carcinoma from its various mimickers in liver magnetic resonance imaging: what are the tips when using hepatocyte-specific agents? World J Gastroenterol 2016;22:284-299.

7. Hope TA, Fowler KJ, Sirlin CB, Costa EA, Yee J, Yeh BM, et al. Hepatobiliary agents and their role in LI-RADS. Abdom Imaging 2015;40:613-625.

8. Omata M, Cheng AL, Kokudo N, Kudo M, Lee JM, Jia J, et al. AsiaPacific clinical practice guidelines on the management of hepatocelIular carcinoma: a 2017 update. Hepatol Int 2017;11:317-370.

9. Kudo M, Kitano M, Sakurai T, Nishida N. General rules for the clinical and pathological study of primary liver cancer, nationwide follow-up survey and clinical practice guidelines: the outstanding achievements of the liver cancer study group of Japan. Dig Dis 2015;33:765-770.

10. Korean Liver Cancer Study Group (KLCSG); National Cancer Center, Korea (NCC). 2014 KLCSG-NCC Korea practice guideline for the management of hepatocellular carcinoma. Gut Liver 2015;9:267317.

11. Mitchell DG, Bruix J, Sherman M, Sirlin CB. LI-RADS (Liver Imaging Reporting and Data System): summary, discussion, and consensus of the LI-RADS Management Working Group and future directions. Hepatology 2015;61:1056-1065.

12. Renzulli M, Biselli M, Brocchi S, Granito A, Vasuri F, Tovoli F, et al. New hallmark of hepatocellular carcinoma, early hepatocellular carcinoma and high-grade dysplastic nodules on Gd-EOB-DTPA MRI in patients with cirrhosis: a new diagnostic algorithm. Gut 2018;67:1674-1682.
13. Choi SH, Byun JH, Lim YS, Yu E, Lee SJ, Kim SY, et al. Diagnostic criteria for hepatocellular carcinoma $<=3 \mathrm{~cm}$ with hepatocytespecific contrast-enhanced magnetic resonance imaging. J Hepatol 2016:64:1099-1107.

14. Joo I, Lee JM, Lee DH, Jeon JH, Han JK. Retrospective validation of a new diagnostic criterion for hepatocellular carcinoma on gadoxetic acid-enhanced MRI: can hypointensity on the hepatobiliary phase be used as an alternative to washout with the aid of ancillary features? Eur Radiol 2019;29:1724-1732.

15. Kim YY, An C, Kim S, Kim MJ. Diagnostic accuracy of prospective application of the Liver Imaging Reporting and Data System (LI-RADS) in gadoxetate-enhanced MRI. Eur Radiol 2018;28:2038-2046.

16. Choi SH, Byun JH, Kim SY, Lee SJ, Won HJ, Shin YM, et al. Liver Imaging Reporting and Data System v2014 with gadoxetate disodiumenhanced magnetic resonance imaging: validation of LI-RADS category 4 and 5 criteria. Invest Radiol 2016;51:483-490.

17. Kierans AS, Kang SK, Rosenkrantz AB. The diagnostic performance of dynamic contrast-enhanced MR imaging for detection of small hepatocellular carcinoma measuring up to $2 \mathrm{~cm}$ : a meta-analysis. Radiology 2016;278:82-94.

18. Chen L, Zhang L, Bao J, Zhang J, Li C, Xia Y, et al. Comparison of MRI with liver-specific contrast agents and multidetector row CT for the detection of hepatocellular carcinoma: a meta-analysis of 15 direct comparative studies. Gut 2013;62:1520-1521.

19. Wu LM, Xu JR, Gu HY, Hua J, Chen J, Zhu J, et al. Is liver-specific gadoxetic acid-enhanced magnetic resonance imaging a reliable tool for detection of hepatocellular carcinoma in patients with chronic liver disease? Dig Dis Sci 2013;58:3313-3325.

20. Park MJ, Kim YK, Lee MW, Lee WJ, Kim YS, Kim SH, et al. Small hepatocellular carcinomas: improved sensitivity by combining gadoxetic acid-enhanced and diffusion-weighted MR imaging patterns. Radiology 2012;264:761-770.

21. Kim HD, Lim YS, Han S, An J, Kim GA, Kim SY, et al. Evaluation of early-stage hepatocellular carcinoma by magnetic resonance imaging with gadoxetic acid detects additional lesions and increases overall survival. Gastroenterology 2015;148:1371-1382.

22. Kim BR, Lee JM, Lee DH, Yoon JH, Hur BY, Suh KS, et al. Diagnostic performance of gadoxetic acid-enhanced liver MR imaging versus multidetector CT in the detection of dysplastic nodules and early hepatocellular carcinoma. Radiology 2017;285:134-146.

23. Park YN. Update on precursor and early lesions of hepatocellular carcinomas. Arch Pathol Lab Med 2011;135:704-715.

24. Choi JY, Lee JM, Sirlin CB. CT and MR imaging diagnosis and staging of hepatocellular carcinoma: part I. Development, growth, and spread: key pathologic and imaging aspects. Radiology 2014;272:635-654

25. Ueno A, Masugi Y, Yamazaki K, Komuta M, Effendi K, Tanami Y, et al. OATP1B3 expression is strongly associated with $\mathrm{Wnt} / \mathrm{\beta}$-catenin 
signalling and represents the transporter of gadoxetic acid in hepatocellular carcinoma. J Hepatol 2014;61:1080-1087.

26. Yoneda N, Matsui O, Kitao A, Kozaka K, Kobayashi S, Sasaki M, et al. Benign hepatocellular nodules: hepatobiliary phase of gadoxetic acid-enhanced MR imaging based on molecular background. Radiographics 2016;36:2010-2027.

27. Kitao A, Matsui O, Yoneda N, Kozaka K, Shinmura R, Koda W, et al. The uptake transporter OATP8 expression decreases during multistep hepatocarcinogenesis: correlation with gadoxetic acid enhanced MR imaging. Eur Radiol 2011;21:2056-2066.

28. Suh CH, Kim KW, Pyo J, Lee J, Kim SY, Park SH. Hypervascular transformation of hypovascular hypointense nodules in the hepatobiliary phase of gadoxetic acid-enhanced MRI: a systematic review and meta-analysis. AJR Am J Roentgenol 2017;209:781-789.

29. Toyoda H, Kumada T, Tada T, Niinomi T, Ito T, Sone $Y$, et al. Nonhypervascular hypointense nodules detected by Gd-EOB-DTPAenhanced MRI are a risk factor for recurrence of HCC after hepatectomy. J Hepatol 2013;58:1174-1180.

30. Lee DH, Lee JM, Lee JY, Kim SH, Kim JH, Yoon JH, et al. Non-hypervascular hepatobiliary phase hypointense nodules on gadoxetic acid-enhanced MRI: risk of HCC recurrence after radiofrequency ablation. J Hepatol 2015;62:1122-1130.

31. Korean Society of Abdominal Radiology. Diagnosis of hepatocellular carcinoma with gadoxetic acid-enhanced MRI: 2016 consensus recommendations of the Korean Society of Abdominal Radiology. Korean J Radiol 2017;18:427-443.

32. Joo I, Lee JM, Lee DH, Jeon JH, Han JK, Choi BI. Noninvasive diagnosis of hepatocellular carcinoma on gadoxetic acid-enhanced MRI: can hypointensity on the hepatobiliary phase be used as an alternative to washout? Eur Radiol 2015;25:2859-2868.

33. Choi SH, Lee SS, Kim SY, Park SH, Park SH, Kim KM, et al. Intrahepatic cholangiocarcinoma in patients with cirrhosis: differentiation from hepatocellular carcinoma by using gadoxetic acid-enhanced MR imaging and dynamic CT. Radiology 2017;282:771-781.

34. Kim R, Lee JM, Shin Cl, Lee ES, Yoon JH, Joo I, et al. Differentiation of intrahepatic mass-forming cholangiocarcinoma from hepatocellular carcinoma on gadoxetic acid-enhanced liver MR imaging. Eur Radiol 2016;26:1808-1817.

35. American College of Radiology (ACR). CT/MRI LI-RADS(R) v2018. ACR web site, <https://www.acr.org/Clinical-Resources/Reportingand-Data-Systems/LI-RADS/CT-MRI-LI-RADS-v2018>. Accessed 17 Nov 2018

36. Lee DH, Lee JM, Baek JH, Shin Cl, Han JK, Choi BI. Diagnostic performance of gadoxetic acid-enhanced liver MR imaging in the detection of HCCS and allocation of transplant recipients on the basis of the Milan criteria and UNOS guidelines: correlation with histopathologic findings. Radiology 2015;274:149-160.

37. Min JH, Kim JM, Kim YK, Kang TW, Lee SJ, Choi GS, et al. Prospec- tive intraindividual comparison of magnetic resonance imaging with gadoxetic acid and extracellular contrast for diagnosis of hepatocellular carcinomas using the Liver Imaging Reporting and Data System. Hepatology 2018;68:2254-2266.

38. Chong YS, Kim YK, Lee MW, Kim SH, Lee WJ, Rhim HC, et al. Differentiating mass-forming intrahepatic cholangiocarcinoma from atypical hepatocellular carcinoma using gadoxetic acid-enhanced MRI. Clin Radiol 2012;67:766-773.

39. Joo I, Lee JM, Lee SM, Lee JS, Park JY, Han JK. Diagnostic accuracy of liver imaging reporting and data system (LI-RADS) v2014 for intrahepatic mass-forming cholangiocarcinomas in patients with chronic liver disease on gadoxetic acid-enhanced MRI. J Magn Reson Imaging 2016:44:1330-1338.

40. Jeong HT, Kim MJ, Chung YE, Choi JY, Park YN, Kim KW. Gadoxetate disodium-enhanced MRI of mass-forming intrahepatic cholangiocarcinomas: imaging-histologic correlation. AJR Am J Roentgenol 2013;201:W603-W611.

41. Min JH, Kim YK, Choi SY, Jeong WK, Lee WJ, Ha SY, et al. Differentiation between cholangiocarcinoma and hepatocellular carcinoma with target sign on diffusion-weighted imaging and hepatobiliary phase gadoxetic acid-enhanced MR imaging: classification tree analysis applying capsule and septum. Eur J Radiol 2017;92:1-10.

42. Hwang J, Kim YK, Min JH, Choi SY, Jeong WK, Hong SS, et al. Capsule, septum, and $\mathrm{T} 2$ hyperintense foci for differentiation between large hepatocellular carcinoma $(\geq 5 \mathrm{~cm}$ ) and intrahepatic cholangiocarcinoma on gadoxetic acid MRI. Eur Radiol 2017;27:4581-4590.

43. Huang B, Wu L, Lu XY, Xu F, Liu CF, Shen WF, et al. Small intrahepatic cholangiocarcinoma and hepatocellular carcinoma in cirrhotic livers may share similar enhancement patterns at multiphase dynamic MR imaging. Radiology 2016;281:150-157.

44. Aishima S, Oda Y. Pathogenesis and classification of intrahepatic cholangiocarcinoma: different characters of perihilar large duct type versus peripheral small duct type. J Hepatobiliary Pancreat Sci 2015;22:94-100.

45. Xu J, Igarashi S, Sasaki M, Matsubara T, Yoneda N, Kozaka K, et al. Intrahepatic cholangiocarcinomas in cirrhosis are hypervascular in comparison with those in normal livers. Liver Int 2012;32:11561164.

46. Joo I, Lee JM, Yoon JH. Imaging diagnosis of intrahepatic and perihilar cholangiocarcinoma: recent advances and challenges. Radiology 2018;288:7-13.

47. Brunt E, Aishima S, Clavien PA, Fowler K, Goodman Z, Gores G, et al. CHCC-CCA: consensus terminology for primary liver carcinomas with both hepatocytic and cholangiocytic differentation. Hepatology 2018;68:113-126.

48. Hwang J, Kim YK, Park MJ, Lee MH, Kim SH, Lee WJ, et al. Differentiating combined hepatocellular and cholangiocarcinoma from mass-forming intrahepatic cholangiocarcinoma using gadoxetic 
Yeun-Yoon Kim, et al. EOB-MRI: HCC and mimickers

acid-enhanced MRI. J Magn Reson Imaging 2012;36:881-889.

49. Park SH, Lee SS, Yu E, Kang HJ, Park Y, Kim SY, et al. Combined hepatocellular-cholangiocarcinoma: gadoxetic acid-enhanced MRI findings correlated with pathologic features and prognosis. J Magn Reson Imaging 2017;46:267-280.

50. Jeon SK, Joo I, Lee DH, Lee SM, Kang HJ, Lee KB, et al. Combined hepatocellular cholangiocarcinoma: LI-RADS v2017 categorisation for differential diagnosis and prognostication on gadoxetic acidenhanced MR imaging. Eur Radiol 2019;29:373-382.

51. Silva $A C$, Evans JM, McCullough $A E$, Jatoi MA, Vargas HE, Hara AK. MR imaging of hypervascular liver masses: a review of current techniques. Radiographics 2009;29:385-402.

52. Kitao A, Zen Y, Matsui O, Gabata T, Kobayashi S, Koda W, et al. Hepatocellular carcinoma: signal intensity at gadoxetic acid-enhanced MR imaging--correlation with molecular transporters and histopathologic features. Radiology 2010;256:817-826.

53. Suh YJ, Kim MJ, Choi JY, Park YN, Park MS, Kim KW. Differentiation of hepatic hyperintense lesions seen on gadoxetic acid-enhanced hepatobiliary phase MRI. AJR Am J Roentgenol 2011;197:W44-W52.

54. Kitao A, Matsui O, Yoneda N, Kita R, Kozaka K, Kobayashi S, et al. Differentiation between hepatocellular carcinoma showing hyperintensity on the hepatobiliary phase of gadoxetic acid-enhanced MRI and focal nodular hyperplasia by CT and MRI. AJR Am J Roentgenol 2018;211:347-357.

55. Nault JC, Paradis V, Cherqui D, Vilgrain V, Zucman-Rossi J. Molecular classification of hepatocellular adenoma in clinical practice. J Hepatol 2017;67:1074-1083.

56. Ba-Ssalamah A, Antunes C, Feier D, Bastati N, Hodge JC, Stift $J$, et al. Morphologic and molecular features of hepatocellular adenoma with gadoxetic acid-enhanced MR imaging. Radiology 2015;277:104-113.

57. Doo KW, Lee CH, Choi JW, Lee J, Kim KA, Park CM. "Pseudo washout" sign in high-flow hepatic hemangioma on gadoxetic acid contrast-enhanced MRI mimicking hypervascular tumor. AJR Am J Roentgenol 2009;193:W490-W496.

58. Kim B, Byun JH, Kim HJ, Won HJ, Kim SY, Shin YM, et al. Enhance- ment patterns and pseudo-washout of hepatic haemangiomas on gadoxetate disodium-enhanced liver MRI. Eur Radiol 2016;26:191198.

59. Guglielmo FF, Mitchell DG, Roth CG, Deshmukh S. Hepatic MR imaging techniques, optimization, and artifacts. Magn Reson Imaging Clin N Am 2014;22:263-282

60. Tamada T, Ito K, Yamamoto A, Sone T, Kanki A, Tanaka F, et al. Hepatic hemangiomas: evaluation of enhancement patterns at dynamic MRI with gadoxetate disodium. AJR Am J Roentgenol 2011;196:824830.

61. Nam SJ, Yu JS, Cho ES, Kim JH, Chung JJ. High-flow haemangiomas versus hypervascular hepatocellular carcinoma showing "pseudowashout" on gadoxetic acid-enhanced hepatic MRI: value of diffusion-weighted imaging in the differential diagnosis of small lesions. Clin Radiol 2017;72:247-254.

62. Choi JY, Cho HC, Sun M, Kim HC, Sirlin CB. Indeterminate observations (liver imaging reporting and data system category 3) on MRI in the cirrhotic liver: fate and clinical implications. AJR Am J Roentgenol 2013;201:993-1001.

63. Motosugi U, Ichikawa T, Sou H, Sano K, Tominaga L, Muhi A, et al. Distinguishing hypervascular pseudolesions of the liver from hypervascular hepatocellular carcinomas with gadoxetic acid-enhanced MR imaging. Radiology 2010;256:151-158.

64. Sun HY, Lee JM, Shin Cl, Lee DH, Moon SK, Kim KW, et al. Gadoxetic acid-enhanced magnetic resonance imaging for differentiating small hepatocellular carcinomas ( $<$ or $=2 \mathrm{~cm}$ in diameter) from arterial enhancing pseudolesions: special emphasis on hepatobiliary phase imaging. Invest Radiol 2010;45:96-103.

65. Lee SJ, Kim SY, Kim KW, Kim JH, Kim HJ, Lee MG, et al. Hepatic angiomyolipoma versus hepatocellular carcinoma in the noncirrhotic liver on gadoxetic acid-enhanced MRI: a diagnostic challenge. AJR Am J Roentgenol 2016;207:562-570.

66. Kim R, Lee JM, Joo I, Lee DH, Woo S, Han JK, et al. Differentiation of lipid poor angiomyolipoma from hepatocellular carcinoma on gadoxetic acid-enhanced liver MR imaging. Abdom Imaging 2015;40:531541. 